\section{MINERAL PROCESSING}

\section{Proceedings of the Second United Nations Inter- national Confarence on the Peaceful Uses of Atomic Energy}

Held in Geneva, 1 September-13 September, 1958. Vol. 3: Processing of Raw Materials. Pp. ix +607. (Geneva : United Nations; London: H.M. Stationery Office, 1958.) 64 Swiss francs ; 1068. ; 15 dollars.

HIS volume is a collection of seventy-eight papers, each dealing with some aspect of the technology of winning and processing the ores of metals of interest in the field of atomic energy. A group of three papers deals with the methods of mining used for obtaining uranium ores in North Americe. Five are concerned with mathematical aspects of evaluating ore deposits and nine with instrumental methods of detecting and estimating radioactive materials, including potassium salts. The rest of the volume is concerned almost entirely with chemical aspects of mineral processing and physical methods of analysis for uranium, thorium and beryllium. There is also a valuable review of the chemical methods available for estimating uranium.

Very little work is reported on physical methods of ore concentration, so presumably the conventional techniques have proved adequate. An automatic method of sorting uranium-bearing rock using the radioactivity of desirable pieces to identify them is described, however, and there is an account of a normal type of process for separating the constituents of a beach sand containing monazite. There has also been limited interest in flotation as a means of concentrating minerals relevant to the Conference except in special cases. One of these concerns a lode deposit of monazite in South Africa which could not be treated by electrostatic and magnetic methods and another is, of course, the difficult problem of the separation of beryl from the other silicates which accompany it in Nature. The paper dealing with the latter topic shows with particular emphasis that valuable as the information presented at this Conference may be, there is much that is not given. Thus a large amount of data on the use of carboxylic acids, such as oleic acid, for separating beryl is given, followed by a table showing very much better results. These are said to have been obtrined using a "special procedure"; but whether this means that the same reagents were used in a different way or that other reagents are better than those described in the paper is not clear.

Pretreatment of uranium ores before dissolving out the uranium has not been necessary in most cases, and is scarcely likely to be economically possible in the near future. However, a large amount of the known potential uranium reserves occurs as very low-grade ore, and three papers deal at least in part with the flotation of uranium minerals. The most active field of work in uranium mineral processing has undoubtedly been the study of methods for dissolving the uranium and recovering it from the resulting aqueous solution. Leaching either with acid or cerbonate-bicarbonate mixtures has received about equal attention, and although the chemistry of both processes is well understood there are some interesting accounts of their application to particular ores. The work designed to produce an oxidizing sulphuric acid solution suitable for use as a leaching reagent by oxidation of sulphur dioxide in the presence of manganese or ferric ions as catalyst is worthy of particular attention.

Recovery of uranium from solution is carried out by solvent extraction or ion exchange, and there is a comparison of the relative merits of the two processes in various circumstances. Considerable interest in the treatment of unclarified solutions using the resin-in-pulp method or solvent extraction of the slurry is shown by the number of papers on these subjects. This is due to the difficulty of removing the suspended solids, and one communication deals with the use of flocculating agents to assist in this process. Of interest also is the method of reducing uranium in solution to precipitate the dioxide, using hydrogen with nickel or uranium dioxide as catalyst.

Chemical methods of cracking minerals are not confined to uranium treatment, of course, but few other cases are dealt with. There are, however, papers dealing with the breaking down of thorium minerals and of zircon.

It is unfortunate that the volume contains no index and that few of the papers include a summary. In spite of this, however, the book should prove valuable to anyone wishing to gain an idea of recent advances in the hydrometallurgical prosessing of minerals.

A. R. Burkin

\section{ELECTROMAGNETISM AND RELA- TIVITY \\ Les Principes de la Théorie Électromagnétique et de la Relativité}

Par Prof. Marie-Antoinette Tonnelat. Pp. 394. (Paris:- Masson et Cie., 1959.) 5,000 francs.

HIS text-book is divided into three parts, dealing with electromagnetism, special relativity and general relativity. The section on special relativity is the largest and the most complete part of the book. It sets out the historical development of the ideas and concepts which led, in the end, to the special theory, and it traces very carefully the interplay between the theoretical concepts and the few decisive experiments during the course of this development.

The main factors on the theoretical side were very straightforward. First, Newton's equations of motion and the Galilean invariance which resulted implied that no (inertial) reference system had preference over any other system moving uniformly relative to the first. Then, later, the nineteenth-century ideas on the propagation of light appeared to single out one absolute reference system, which was specified by the ether. Later again, it was seen that Maxwell's equations were not Galilean invariant, and therefore they, too, supported the idea of the absolute reference system. Thus it seemed that all that was needed to determine absolute motion was a few accurate optical experiments. However, the culmination of these experiments was the famous interferometer work of Michelson and Morley, which gave the nul result.

This story is well told, with adequate digressions to deal with related matters of interest. For example, the relation is pointed out between the experiments of Fizeau, Hoek, and others on the propagation of light in moving media, and the experiment of $\mathbf{H}$. $\mathbf{A}$. Wilson on the effects of moving a dielectric in a magnetic field. In addition, the mathematical appara- 